\title{
Schur Complement of con-s-k-EP Matrices
}

\author{
Bagyalakshmi Karuna Nithi Muthugobal \\ Ramanujan Research Centre, Department of Mathematics, Government Arts College (Autonomous), Kumbakonam, India \\ Email: bkn.math@gmail.com
}

Received February 8, 2012; revised March 8, 2012; accepted March 15, 2012

\begin{abstract}
Necessary and sufficient conditions for a schur complement of a con-s-k-EP matrix to be con-s-k-EP are determined.

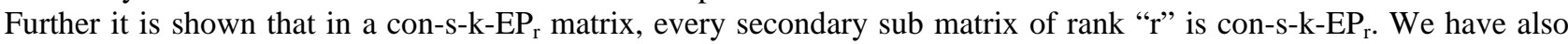
discussed the way of expressing a matrix of rank $r$ as a product of con-s-k-E $P_{r}$ matrices. Necessary and sufficient conditions for products of con-s-k-EP $P_{r}$ partitioned matrices to be con-s-k-EP $P_{r}$ are given.
\end{abstract}

Keywords: con-s-k-EP Matrices; Partitioned Matrices; Schur Complements

\section{Introduction}

Let $C_{n \times n}$ be the space of $n \times n$ complex matrices of order $n$. Let $\mathrm{C}_{\mathrm{n}}$ be the space of all complex n-tuples. For $A \in C_{\mathrm{n} \times \mathrm{n}}$, let $\bar{A}, A^{T}, A^{*}, A^{S}, \bar{A}^{S}, A^{\dagger}, R(A), N(A)$ and $\rho(A)$ denote the conjugate, transpose, conjugate transpose, secondary transpose, conjugate secondary transpose, Moore-Penrose inverse, range space, null space and rank of $A$, respectively. $A$ solution $X$ of the equation $A X A=A$ is called generalized inverses of $\mathrm{A}$ and is denoted by $A^{-}$. If $A \in C_{\mathrm{n} \times \mathrm{n}}$, then the unique solution of the equations $A X A$ $=A, X A X=X,[A X]^{*}=A X,[X A]^{*}=X A[2]$ is called the moore penrose inverse of $A$ and is denoted by $A^{\dagger}$.

A matrix $A$ is called con-s-k-EP $\mathrm{E}_{\mathrm{r}}$ if $\rho(A)=\mathrm{r}$ and $N(A)=N\left(A^{T} V K\right)$ or $R(A)=R\left(K V A^{T}\right)$. Throughout this paper let " $k$ " be the fixed product of disjoint transposition in $S_{\mathrm{n}}=\{1,2, \cdots, \mathrm{n}\}$ and $\mathrm{K}$ be the associated permute- tion matrix. Let us define the function $k(x)=\left(x_{k(l)}, x_{k(2)}, \cdots, x_{k(n)}\right)$. A matrix $A=\left(\mathrm{a}_{\mathrm{ij}}\right) \in C_{\mathrm{nxn}}$ is s-k symmetric if $a_{i j}=a_{n-k(j)+1, n-k(i)+1}$ for $\mathrm{i}, \mathrm{j}=1,2, \cdots, \mathrm{n}$. A matrix $A \in C_{\mathrm{nxn}}$ is said to be con-s-k-EP if it satisfies the condition $A x=0 \Leftrightarrow A^{s} k(x)=0$ or equivalently $N(A)$ $=N\left(A^{T} V K\right)$. In addition to that $\mathrm{A}$ is con-s-k-EP $\Leftrightarrow K V A$ is con-EP or $A V K$ is con-EP and $\mathrm{A}$ is con-s-k-EP $\Leftrightarrow A^{T}$ is con-s-k-EP. Moreover $A$ is said to be con-s-k-EP $\mathrm{EP}_{\mathrm{r}}$ if con-s-k-EP and of rank r. For further properties of con-s-k-EP matrices one may refer [1].

In this paper we derive the necessary and sufficient conditions for a schur complement of a con-s-k-EP matrix to be con-s-k-EP. Further it is shown that in a con$s-k-E P_{r}$ matrix, every secondary submatrix of rank $r$ is con-s-k-EP $P_{r}$. We have also discussed the way of expressing a matrix of rank $r$ as a product of con-s-k-E $\mathrm{P}_{\mathrm{r}}$ matrices. Necessary and sufficient conditions for products of con-s-k-EP $\mathrm{r}_{\mathrm{r}}$ partitioned matrices to be con-s-k-EP $\mathrm{P}_{\mathrm{r}}$ are given. In this sequel, we need the following theorems.

\section{Theorem 1.1 [2]}

Let $\mathrm{A}, \mathrm{B} \in \mathrm{C}_{\mathrm{nxn}}$, then

1) $N(A) \subseteq N(B) \Leftrightarrow R\left(B^{T}\right) \subseteq R\left(A^{T}\right) \Leftrightarrow B=B A^{-} A$ for all $A^{-} \in A\{1\}$

2) $N\left(A^{T}\right) \subseteq N\left(B^{T}\right) \Leftrightarrow R(B) \subseteq R(A) \Leftrightarrow B=A A^{-} B$ for all $A^{-} \in A\{1\}$

\section{Theorem 1.2 [3]}

Let, $M=\left[\begin{array}{ll}A & B \\ C & D\end{array}\right]$, then

$M^{\dagger}=\left[\begin{array}{cc}A^{\dagger}+A^{\dagger} B(M / A)^{\dagger} C A & -A^{\dagger} B(M / A)^{\dagger} \\ -(M / A)^{\dagger} C A^{\dagger} & (M / A)^{\dagger}\end{array}\right]$

$\Leftrightarrow N(A) \subseteq N(C), N\left(A^{T}\right) \subseteq N\left(B^{T}\right)$,

$N(M / A)^{T} \subseteq N\left(C^{T}\right)$ and $N(M / A) \subseteq N(B)$.

Also, $M^{\dagger}=\left[\begin{array}{cc}(M / D)^{\dagger} & -A^{\dagger} B(M / A)^{\dagger} \\ -D^{\dagger} C(M / D)^{\dagger} & (M / A)^{\dagger}\end{array}\right]$

$\Leftrightarrow N(A) \subseteq N(C)$

$N\left(A^{T}\right) \subseteq N\left(B^{T}\right), N(M / A)^{T} \subseteq N\left(C^{T}\right)$,

$N(M / A) \subseteq N(B)$ and $\Leftrightarrow N(D) \subseteq N(B)$,

$N\left(D^{T}\right) \subseteq N\left(C^{T}\right), N(M / D)^{T} \subseteq N\left(B^{T}\right)$,

$N(M / D) \subseteq N(C)$.

When $\rho(M)=\rho(A)$, then $M=\left(\begin{array}{cc}A & B \\ C & C A^{-} B\end{array}\right)$ and $M=\left(\begin{array}{ll}A^{T} P A^{T} & A^{T} P C^{T} \\ B^{T} P A^{T} & B^{T} P C^{T}\end{array}\right)$, 
where, $P=\left(A A^{T}+B B^{T}\right)^{-} A\left(A^{T} A+C^{T} C\right)^{-}$.

Theorem 1.3 [4]

Let $A, B \in C_{n \times n}$ and $U \in C_{n \times n}$ be any nonsingular matrix, then,

1) $R(A)=R(B) \Leftrightarrow R(U A U)^{T}=R(U B U)^{T}$

2) $N(A)=N(B) \Leftrightarrow N(U A U)^{T}=N(U B U)^{T}$

\section{Schur Complements of con-s-k-EP Matrices}

In this section we consider a $2 \mathrm{r} \times 2 \mathrm{r}$ matrix $M$ Partitioned in the form,

$$
M=\left(\begin{array}{ll}
A & B \\
C & D
\end{array}\right)
$$

where $A, B, C$ and $D$ are all square matrices. If a partitioned matrix $M$ of the form 2.1 is con-s-k-EP, then in general, the schur complement of $C$ in $M$, that is $(M / C)$ is not con-s-k-EP. Here, necessary and sufficient conditions for $(M / C)$ to be con-s-k-EP are obtained for the class $\rho(M)=\rho(C)$ and $\rho(M) \neq \rho(C)$, analogous to that of results in [5]. Now we consider the matrix

$$
S=\left(\begin{array}{ll}
(M / A) & (M / B) \\
(M / C) & (M / B)
\end{array}\right)
$$

the matrix formed by the Schur complements of $M$ over $A, B, C$ and $D$ respectively. This is also a partitioned matrix. If a partitioned matrix $S$ of the form 2.2 is con-s$\mathrm{k}$-EP, then in general, Schur complement of $(M / C)$ in $S$, that is $[S /(M / C)]$ is not con-s-k-EP. Here, the necessary and sufficient conditions for $[S /(M / C)]$ to be con-s-k-EP are obtained for the class $\rho(S)=\rho(M / C)$ and $\rho(S) \neq \rho(M / C)$, analogous to that of results in [5]

As an application, a decomposition of a partitioned matrix into a sum of con-s-k-EP $\mathrm{P}_{\mathrm{r}}$ matrices is obtained. Further it is shown that in a con-s-k-EP $P_{r}$ matrix, every secondary sub matrix of rank $r$, is con-s-k-EP. Throughout this section let $\mathrm{k}=\mathrm{k}_{1} \mathrm{k}_{2}$ with.

$$
K=\left(\begin{array}{cc}
K_{1} & 0 \\
0 & K_{2}
\end{array}\right)
$$

where $K_{1}$ and $K_{2}$ are the permutation matrices relative to $\mathrm{k}_{1}$ and $\mathrm{k}_{2}$ and let " $V$ " be the permutation matrix with units in its secondary diagonal of order $2 \mathrm{r} \times 2 \mathrm{r}$ partitioned in such a way that

$$
V=\left(\begin{array}{ll}
0 & v \\
v & 0
\end{array}\right)
$$

\section{Theorem 2.5}

Let $S$ be a matrix of the form 2.2 with $\mathrm{N}(M / C) \subseteq \mathrm{N}(M / A)$ and $\mathrm{N}[\mathrm{S} /(M / C)] \subseteq \mathrm{N}(M / D)$, then the following are equivalent:

1) $S$ is a con-s-k-EP $P_{\mathrm{r}}$ matrix with $\mathrm{k}=\mathrm{k}_{1} \mathrm{k}_{2}$ and $\mathrm{V}=\left(\begin{array}{ll}0 & v \\ v & 0\end{array}\right)$.

2) $(M / C)$ is a con-s-k-EP, $[S /(M / C)]$ is con-s-k $-\mathrm{EP}$.

$N(M / C)^{T} \subseteq N(M / D)^{T}$ and

$N[S /(M / C)]^{T} \subseteq N(M / A)^{T}$.

3) Both the matrices

$\left(\begin{array}{cc}(M / C) & 0 \\ (M / A) & {[S /(M / C)]}\end{array}\right)$ and $\left(\begin{array}{cc}(M / C) & (M / D) \\ 0 & {[S /(M / C)]}\end{array}\right)$

are con-s-k-EP .

\section{Proof:}

Since $S$ is con-s-k-EP $P_{\mathrm{r}}$ with $\mathrm{k}=\mathrm{k}_{1} \mathrm{k}_{2}$, KVS is Con-EP and $K=\left(\begin{array}{cc}K_{1} & o \\ o & K_{2}\end{array}\right)$ where $K_{1}$ and $K_{2}$ are permutation matrices associated with $\mathrm{k}_{1}$ and $\mathrm{k}_{2}$ and $V=\left(\begin{array}{ll}o & v \\ v & o\end{array}\right)$.

Consider $P=\left(\begin{array}{cc}I & (M / A)(M / C)^{-} \\ O & I\end{array}\right)$,

$Q=\left(\begin{array}{cc}I & O \\ (M / D)[S /(M / C)]^{-} & I\end{array}\right)$ and

$L=\left(\begin{array}{cc}O & {[S /(M / C)]} \\ (M / C) & O\end{array}\right)$.

Clearly $\mathrm{P}$ and $\mathrm{Q}$ are non singular.

Now,

$$
\begin{aligned}
& K V P Q L=\left(\begin{array}{cc}
K_{1} & O \\
O & K_{2}
\end{array}\right)\left(\begin{array}{ll}
O & v \\
v & O
\end{array}\right)\left(\begin{array}{cc}
I & (M / A)(M / C)^{-} \\
O & I
\end{array}\right)\left(\begin{array}{cc}
I & O \\
(M / D)[S /(M / C)]^{-} & I
\end{array}\right)\left(\begin{array}{cc}
O & {[S /(M / C)]} \\
(M / C) & O
\end{array}\right) \\
& =\left(\begin{array}{cc}
O & K_{1} v \\
K_{2} v & O
\end{array}\right)\left(\begin{array}{cc}
I+(M / A)(M / C)^{-}(M / D)[S /(M / C)]^{-} & (M / A)(M / C)^{-} \\
(M / D)[S /(M / C)]^{-} & I
\end{array}\right)\left(\begin{array}{cc}
O & {[S /(M / C)]} \\
(M / C) & O
\end{array}\right) \\
& =\left(\begin{array}{cc}
K_{1} v(M / C) & K_{1} v(M / D)[S /(M / C)]^{-}[S /(M / C)] \\
K_{2} v(M / A)(M / C)^{-}(M / C) & K_{2} v[S /(M / C)]+(M / A)(M / C)^{-}(M / D)[S /(M / C)]^{-}[S /(M / C)]
\end{array}\right)
\end{aligned}
$$


Since, $N(M / C) \subseteq N(M / A)$, by Theorem 1.1 we have $(M / A)=(M / A)(M / C)^{-}(M / C)$,

that is, $K_{2} v(M / A)=K_{2} v(M / A)(M / C)^{-}(M / C)$.

Since, $N[S /(M / C)] \subseteq N /(M / D)$,

we have by Theorem 1.1

$(M / D)=(M / D)[S /(M / C)]^{-}[S /(M / C)]$.

That is,

$K_{1} v(M / D)=K_{1} v(M / D)[S /(M / C)]^{-}[S /(M / C)]$.

Also,

$$
\begin{aligned}
& K_{2} v[S /(M / C)] \\
& +(M / A)(M / C)^{-}(M / D)[S /(M / C)]^{-}[S /(M / C)] \\
& =K_{2} v(M / B) .
\end{aligned}
$$

Since,

$\left([S /(M / C)]=(M / B)-(M / A)(M / C)^{-}(M / D)\right)$,

therefore,

$$
\begin{aligned}
K V P Q L & =\left(\begin{array}{cc}
K_{1} v(M / C) & K_{1} v(M / D) \\
K_{2} v(M / A) & K_{2} v(M / B)
\end{array}\right) \\
& =\left(\begin{array}{cc}
O & K_{1} v \\
K_{2} v & O
\end{array}\right)\left(\begin{array}{ll}
(M / A) & (M / B) \\
(M / C) & (M / D)
\end{array}\right) \\
& =\left(\begin{array}{cc}
K_{1} & O \\
O & K_{2}
\end{array}\right)\left(\begin{array}{ll}
O & v \\
v & O
\end{array}\right)\left(\begin{array}{ll}
(M / A) & (M / B) \\
(M / C) & (M / D)
\end{array}\right) \\
& =K V S
\end{aligned}
$$

Thus $K V S$ is factorized as $K V S=K V P Q L$.

Hence $\rho(K V S)=\rho(L)$ and $N(K V S)=N(L)$.

But $S$ is con-s-k-EP. Therefore, $K V S$ is con-EP (By Theorem 2.11 [1]).

$$
N(K V S)=N(K V S)^{T} \Rightarrow N(L)=N\left(S^{T} V K\right)
$$

Therefore, by using Theorem 1.1 again we get,

$S^{T} V K=S^{T} V K L^{-} L$ holds for every $\mathrm{L}^{-}$.
We choose $L^{-}$as $L^{-}=\left(\begin{array}{cc}O & (M / C)^{-} \\ {[S /(M / C)]^{-}} & O\end{array}\right)$

$$
\begin{aligned}
S^{T} V K & =\left(\begin{array}{ll}
(M / A) & (M / B) \\
(M / C) & (M / D)
\end{array}\right)^{T}\left(\begin{array}{ll}
O & v \\
v & O
\end{array}\right)\left(\begin{array}{cc}
K_{1} & O \\
O & K_{2}
\end{array}\right) \\
& =\left(\begin{array}{ll}
(M / A)^{T} & (M / C)^{T} \\
(M / B)^{T} & (M / D)^{T}
\end{array}\right)^{T}\left(\begin{array}{cc}
O & v K_{2} \\
v K_{1} & O
\end{array}\right) \\
& =\left(\begin{array}{ll}
(M / C)^{T} v K_{1} & (M / A)^{T} v K_{2} \\
(M / D)^{T} v K_{1} & (M / B)^{T} v K_{2}
\end{array}\right)
\end{aligned}
$$

As the equation (at the bottom of this page).

and since

$$
\begin{aligned}
& \rho\left[K_{1} v(M / C)^{T}\right]=\rho\left[K_{1} v(M / C)\right] \\
& \Rightarrow \rho\left[(M / C)^{T} v K_{1}\right]=\rho(M / C) \\
& \Rightarrow N(M / C)=N\left[(M / C)^{T} v K_{1}\right]
\end{aligned}
$$

Hence, $(\mathrm{M} / \mathrm{C})$ is con-s-k-EP.

From $(M / D)^{T} v K_{1}=(M / D)^{T} v K_{1}(M / C)^{-}(M / C)$, is follows that

$$
\begin{aligned}
& N(M / C) \subseteq N\left[(M / D)^{T} v K_{1}\right] \\
& \Rightarrow N\left[(M / C)^{T} v K_{1}\right] \subseteq N\left[(M / D)^{T} v K_{1}\right]
\end{aligned}
$$

(using $(\mathrm{M} / \mathrm{C})$ is con-s-k-EP ).

Therefore $N(M / C)^{T} \subseteq N(M / D)^{T}$.

After substituting

$(M / B)=[S /(M / C)]+(M / A)(M / C)^{-}(M / D)$

and using

$(M / A)^{T} v K_{2}=(M / A)^{T} v K_{2}[S /(M / C)]^{-}[S /(M / C)]$ in

$(M / B)^{T} v K_{2}=(M / B)^{T} v K_{2}[S /(M / C)]^{-}[S /(M / C)]$

$$
\begin{aligned}
& S^{T} V K=S^{T} v K L^{-} L \Rightarrow\left(\begin{array}{ll}
(M / C)^{T} v K_{1} & (M / A)^{T} v K_{2} \\
(M / D)^{T} v K_{1} & (M / B)^{T} v K_{2}
\end{array}\right) \\
& =\left(\begin{array}{cc}
(M / C)^{T} v K_{1} & (M / A)^{T} v K_{2} \\
(M / D)^{T} v K_{1} & (M / B)^{T} v K_{2}
\end{array}\right)\left(\begin{array}{cc}
O & (M / C)^{-} \\
{[S /(M / C)]^{-}} & O
\end{array}\right)\left(\begin{array}{cc}
O & {[S /(M / C)]^{-}} \\
(M / C) & O
\end{array}\right) \\
& =\left(\begin{array}{ll}
(M / C)^{T} v K_{1}(M / C)^{-}(M / C) & A^{T} v K_{2}[S /(M / C)]^{-}[S /(M / C)] \\
(M / D)^{T} v K_{1}(M / C)^{-}(M / C) & B^{T} v K_{2}[S /(M / C)]^{-}[S /(M / C)]
\end{array}\right) \\
& \Rightarrow(M / C)^{T} v K_{1}=(M / C)^{T} v K_{1}(M / C)^{-}(M / C) \\
& \Rightarrow\left[K_{1} v(M / C)\right]^{T}=\left[K_{1} v(M / C)\right]^{T}(M / C)^{-}(M / C) \\
& \Rightarrow N(M / C) \subseteq N\left[K_{1} v(M / C)\right]^{T}=N(M / C)^{T} v K_{1}
\end{aligned}
$$


We get,

$$
\begin{aligned}
& (M / B)^{T} v K_{2}=(M / B)^{T} v K_{2}[S /(M / C)]^{-}[S /(M / C)] \\
& \left([S /(M / C)]+(M / A)(M / C)^{-}(M / B)\right)^{T} v K_{2} \\
& =[S /(M / C)] \\
& +\left[(M / A)(M / C)^{-}(M / B)\right]^{T} v K_{2}[S /(M / C)]^{-}[S /(M / C)] \\
& {[S /(M / C)]^{T} v K_{2}+\left[(M / A)(M / C)^{-}(M / B)\right]^{T} v K_{2}} \\
& =[S /(M / C)]^{T} v K_{2}[S /(M / C)]^{-}[S /(M / C)] \\
& +\left[(M / A)(M / C)^{-}(M / B)\right]^{T} v K_{2}(M / C)^{-}(M / C) \\
& {[S /(M / C)]^{T} v K_{2}} \\
& =[S /(M / C)]^{T} v K_{2}[S /(M / C)]^{-}[S /(M / C)] \\
& \Rightarrow N[S /(M / C)] \subseteq N[S /(M / C)]^{T} v K_{2}
\end{aligned}
$$

By Theorem 1.1 and since

$$
\rho\left(K_{2} v[S /(M / C)]^{T}\right)=\rho[S /(M / C)]^{T}=\rho[S /(M / C)]
$$

we get,

$$
\begin{aligned}
& N\left(K_{2} v[S /(M / C)]^{T}\right)=N[S /(M / C)] \\
& \Rightarrow N\left([S /(M / C)] v K_{2}\right)=N[S /(M / C)] \\
& \Rightarrow[S /(M / C)] \text { is con-s- } \mathrm{k}_{2}-\mathrm{EP}_{\mathrm{r} .}
\end{aligned}
$$

Further

$$
\begin{aligned}
& (M / A)^{T} v K_{2}=(M / A)^{T} v K_{2}[S /(M / C)]^{-}[S /(M / C)] \\
& \Rightarrow N[S /(M / C)] \subseteq N\left((M / A)^{T} v K_{2}\right) \\
& \Rightarrow N\left(K_{2} v[S /(M / C)]^{T}\right) \subseteq N\left((M / A)^{T} v K_{2}\right) \\
& \Rightarrow N\left([S /(M / C)]^{T} v K_{2}\right) \subseteq N\left((M / A)^{T} v K_{2}\right) \\
& \Rightarrow N[S /(M / C)]^{T} \subseteq N(M / A)^{T}
\end{aligned}
$$

Thus 2) holds 2) $\Rightarrow 1$ ). Since $N(M / C) \subseteq N(M / A), N(M / C)^{T} \subseteq N(M / D)^{T}$, $N[S /(M / C)] \subseteq N(M / D)$ and $N[S /(M / C)]^{T} \subseteq N(M / A)^{T}$ holds, according to the assumption by applying Theorem 1.2, $(K V S)^{\dagger}$ is given by the formula

$$
\begin{aligned}
& (K V S)=\left(\begin{array}{cc}
K_{1} v(M / C)+\left(K_{1} v(M / C)\right)\left(K_{1} v(M / D)\right) & \\
\left(K_{2} v[S /(M / C)]^{\dagger}\left(K_{2} v(M / A)\right)\right)\left(K_{1} v(M / C)\right)^{\dagger} & -\left(K_{1} v(M / C)\right)\left(K_{1} v(M / D)\right)\left(K_{2} v[S /(M / C)]^{\dagger}\right) \\
-\left(K_{2} v[S /(M / C)]\right)^{\dagger}\left(K_{2} v(M / A)\right)\left(K_{1} v(M / C)\right)^{\dagger} & K_{2} v[S /(M / C)]^{\dagger}
\end{array}\right)( \\
& =\left(\begin{array}{cc}
K_{1} v(M / C)^{\dagger}+(M / C)^{\dagger}(M / D)[S /(M / C)]^{\dagger}(M / A)\left(K_{1} v(M / C)\right)^{\dagger} & -(M / C)^{\dagger}(M / D)[S /(M / C)]^{\dagger} v K_{2} \\
-[S /(M / C)]^{\dagger}(M / A)\left(K_{1} v(M / C)\right)^{\dagger} & {[S /(M / C)]^{\dagger} v K_{2}}
\end{array}\right) \\
& {[K V S][K V S]^{\dagger}=\left(\begin{array}{cc}
\left(K_{1} v(M / C)\right)\left(K_{1} v(M / C)\right)^{\dagger} & -\left(K_{1} v(M / C)\right)(M / C)^{\dagger} \\
+\left(K_{1} v(M / C)\right)\left((M / C)^{\dagger}(M / D)[S /(M / C)]^{\dagger}\right) & (M / D)[S /(M / C)]^{\dagger \dagger} v K_{2} \\
(M / A)\left(K_{1} v(M / C)\right)^{\dagger}-\left(K_{1} v(M / D)\right)[S /(M / C)]^{\dagger} & +\left(K_{1} v(M / D)\right)[S /(M / C)]^{\dagger} v K_{2} \\
+(M / A)\left(K_{1} v(M / C)\right)^{\dagger} & \\
\left(K_{2} v(M / A)\right)\left(K_{1} v(M / C)\right)^{\dagger}\left(K_{2} v(M / A)\right)(M / C)^{\dagger} & -\left(K_{2} v(M / A)\right)(M / C)^{\dagger} \\
(M / D)[S /(M / C)]^{\dagger}(M / D)\left(K_{1} v(M / C)\right)^{\dagger} & (M / D)[S /(M / C)]^{\dagger} v K_{2} \\
-\left(K_{2} v(M / B)\right)[S /(M / C)]^{\dagger}(M / A)\left(K_{1} v(M / C)\right) & +\left(K_{2} v(M / B)\right)[S /(M / C)] v K_{2}
\end{array}\right)}
\end{aligned}
$$


According to Theorem 1.1 the assumptions $N(M / C) \subseteq$ $N(M / A)$ and $N /(M / C)^{T} \subseteq N /(M / D)^{T} \Rightarrow[S /(M / C)]$ is invariant for every choice of $(M / C)^{-}$

Hence

$$
\begin{aligned}
K_{2} v(M / B) & =K_{2} v[S /(M / C)] \\
& +\left(K_{2} v(M / C)\right)\left(K_{1} v(M / C)^{\dagger}\left(K_{1} v(M / D)\right)\right)
\end{aligned}
$$

Therefore

$$
\begin{aligned}
K_{2} v[S /(M / C)] \\
=K_{2} v(M / B)-\left(K_{2} v(M / A)\right)\left(K_{1} v(M / C)\right)^{\dagger}\left(K_{1} v(M / D)\right) \\
\Rightarrow\left(K_{2} v(M / A)\right)\left(K_{1} v(M / C)\right)^{\dagger}\left(K_{1} v(M / D)\right) \\
\quad=\left(K_{2} v(M / B)\right)-K_{2} v[S /(M / C)] \\
\Rightarrow K_{2} v(M / B)(M / C)^{\dagger}(M / D) \\
\quad=K_{2} v((M / B)-[S /(M / C)]) \\
\Rightarrow(M / A)(M / C)^{\dagger}(M / D)=(M / B)-[S /(M / C)]
\end{aligned}
$$

Further using

$$
\begin{aligned}
& K_{2} v(M / A) \\
& =\left(K_{2} v[S /(M / C)]\right)\left(K_{2} v[S /(M / C)]^{\dagger}\right)\left(K_{2} v(M / A)\right)
\end{aligned}
$$

and

$$
K_{1} v(M / D)=\left(K_{1} v(M / C)\right)\left(K_{1} v(M / C)\right)^{\dagger}\left(K_{1} v(M / D)\right) .
$$

That is

$$
K_{2} v(M / A)
$$$$
=K_{2} v[S /(M / C)][S /(M / C)]^{\dagger} v K_{2} K_{2} v(M / A)
$$$$
=K K_{2} v[S /(M / C)][S /(M / C)]^{\dagger}(M / A)
$$$$
(M / A)=[S /(M / C)][S /(M / C)]^{\dagger}(M / A)
$$

and

$$
K_{1} v(M / D)=K_{1} v(M / C)(M / C)^{\dagger} v K_{1} K_{1} v(M / D)
$$$$
=K_{1} v(M / C)(M / C)^{\dagger}(M / D)
$$$$
(M / D)=(M / C)(M / C)^{\dagger}(M / D),
$$$$
(K V S)(K V S)^{\dagger} \text { reduces to the form, }
$$

As the Equation (a) below.

Again using
$\left(K_{1} v(M / D)\right)^{\dagger}$

$$
=\left(K_{1} v(M / D)\right)\left(K_{2} v[S /(M / C)]\right)^{\dagger}\left(K_{2} v[S /(M / C)]\right)
$$

and

$$
\left(K_{2} v(M / A)\right)=\left(K_{2} v(M / A)\right)\left(K_{1} v(M / C)\right)^{\dagger}\left(K_{1} v(M / C)\right)
$$

that is, $(M / D)=(M / D)[S /(M / C)]^{\dagger}[S /(M / C)]$

and

$(M / A)=(M / A)(M / C)^{\dagger}(M / C),(K V S)(K V S)^{\dagger}$

reduces to the form

As the Equation (b) below.

Since, $(M / C)$ is con-s- $\mathrm{k}_{1}-\mathrm{EP} \Rightarrow K_{1} v(M / C)$ is con-EP.

Therefore we have

$\left[K_{1} v(M / C)\right]\left[K_{1} v(M / C)\right]^{\dagger}$

$=\left[K_{1} v(M / C)\right]^{\dagger}\left[K_{1} v(M / C)\right]$

Similarly, since $[S /(M / C)]$ is con-s- $\mathrm{k}_{2}-\mathrm{EP}_{\mathrm{r}}$. We have,

$$
\begin{aligned}
& \left(K_{2} v(M / C)\right)\left(K_{2} v[S /(M / C)]\right)^{\dagger} \\
& =\left(K_{2} v[S /(M / C)]\right)^{\dagger}\left(K_{1} v[S /(M / C)]\right)
\end{aligned}
$$

Thus

$(K V S)(K V S)^{\dagger}=(K V S)^{\dagger}(K V S)$

$\Rightarrow K V S S^{\dagger} V K=S^{\dagger} V K K V S$

$\Rightarrow K V S S^{\dagger} V K=S^{\dagger} S$

$\Rightarrow K V S S^{\dagger}=S^{\dagger} S K V$

$\Rightarrow S$ is con-s-k-EP (by Theorem 2.11 [1]).

Thus 1) holds 2) $\Leftrightarrow 3$ )

$$
\left(\begin{array}{cc}
K_{2} v(M / C) & 0 \\
K_{2} v(M / A) & K_{2} v[S /(M / C)]
\end{array}\right)
$$

is con-EP if and only if $K_{1} v(M / C)$ and $K_{2} v[S /(M / C)]$ are con-EP.

Therefore,

$$
\left(\begin{array}{cc}
K_{1} & 0 \\
0 & K_{2}
\end{array}\right)\left(\begin{array}{ll}
0 & v \\
v & 0
\end{array}\right)\left(\begin{array}{cc}
(M / C) & 0 \\
(M / A) & {[S /(M / C)]}
\end{array}\right)
$$

$$
\begin{aligned}
& (K V S)(K V S)^{\dagger}=\left(\begin{array}{cc}
\left(K_{1} v(M / C)\right)\left(K_{1} v(M / C)\right)^{\dagger} & 0 \\
0 & \left(K_{2} v[S /(M / C)]\right)\left(K_{2} v[S /(M / C)]\right)^{\dagger}
\end{array}\right) \\
& (K V S)(K V S)^{\dagger}=\left(\begin{array}{cc}
\left(K_{1} v(M / C)\right)\left(K_{1} v(M / C)\right)^{\dagger} & 0 \\
0 & \left(K_{2} v[S /(M / C)]\right)\left(K_{2} v[S /(M / C)]\right)^{\dagger}
\end{array}\right)
\end{aligned}
$$


is con-EP if and only if $K_{1} v(M / C)$ and $K_{2} v(M / C)$ are con-EP.

$\left(\begin{array}{cc}(M / C) & 0 \\ (M / A) & {[S /(M / C)]}\end{array}\right)$ is con-s-k-EP if and only if $(M / C)$ is con-s- $\mathrm{k}_{1}$-EP and $[S /(M / C)]$ is con-s- $\mathrm{k}_{2}$-EP.

Further $N(M / C) \subseteq N(M / A)$

and $N[S /(M / C)]^{T} \subseteq N(M / D)^{T}$

Also $\left(\begin{array}{cc}K_{1} v(M / C) & K_{1} v(M / D) \\ 0 & K_{2} v[S /(M / C)]\end{array}\right)$ is con-EP if and only if and $K_{2} v[S /(M / C)]$ and con-EP.

Therefore, $\left(\begin{array}{cc}(M / C) & (M / D) \\ 0 & {[S /(M / C)]}\end{array}\right)$ is con-s-k-EP if and only if $(M / C)$ is con-s- $\mathrm{k}_{1}$-EP and $[S /(M / C)]$ is con-s- $\mathrm{k}_{2}$-EP further $N(M / C)^{T} \subseteq N(M / D)^{T} \quad$ and $N[S /(M / C)]^{T} \subseteq N(M / D)$.

This proves the equivalence of 2) and 3). The proof is complete.

Theorem 2.7

Let $S$ be a matrix of the form (2.2) with

$N(M / C)^{T} \subseteq N(M / D)^{T}$ and

$N[S /(M / C)]^{T} \subseteq N(M / A)^{T}$, then the following are equivalent.

1) $S$ is con-s-k-EP with $\mathrm{k}=\mathrm{k}_{1} \mathrm{k}_{2}$ where

$K=\left(\begin{array}{cc}K_{1} & 0 \\ 0 & K_{2}\end{array}\right)$ and $V=\left(\begin{array}{cc}0 & v \\ v & 0\end{array}\right)$

2) $(M / C)$ is con-s-k $\mathrm{k}_{1}-\mathrm{EP}$. Further and $[S /(M / C)]$ is con-s-k $\mathrm{k}_{2}$-EP. Further $N(M / C) \subseteq N(M / A)$ and $N[S /(M / C)] \subseteq N(M / D)$

3) Both the matrices $\left(\begin{array}{cc}(M / C) & 0 \\ (M / A) & {[S /(M / C)]}\end{array}\right)$ and $\left(\begin{array}{cc}(M / C) & (M / D) \\ 0 & {[S /(M / C)]}\end{array}\right)$ are con-s-k-EP.

Proof

This follows from Theorem 2.5 and from the fact that $S$ is con-s-k-EP $\Leftrightarrow \mathrm{S}^{\mathrm{T}}$ is con-s-k-EP.

In particular, when $(M / D)=(M / A)^{T}$, we got the following.

Corollary 2.8

$$
\begin{gathered}
\text { Let } S=\left(\begin{array}{ll}
(M / A) & (M / B) \\
(M / C) & (M / A)^{T}
\end{array}\right) \text { with } \\
N(M / C) \subseteq N(M / A) \text { and }
\end{gathered}
$$

$N[S /(M / C)] \subseteq N(M / A)^{T}$.

Then the following are equivalent.

1) $S$ is a con-s-k-EP matrix.

2) $(\mathrm{M} / \mathrm{C})$ is con-s-k $\mathrm{k}_{1}-\mathrm{EP}$ and $[S /(M / C)]$ is con-s$\mathrm{k}_{2}$-EP.

3) The matrix $\left(\begin{array}{cc}(M / C) & 0 \\ (M / A) & {[S /(M / C)]}\end{array}\right)$ is con-s-k- EP.

\section{Remark 2.9}

The conditions taken on $S$ in Theorem 2.6 and Theorem 2.7 are essential. This is illustrated in the following example.

$$
\begin{aligned}
& \text { Let } M=\left(\begin{array}{ll}
A & B \\
C & D
\end{array}\right) \\
& A=\left(\begin{array}{ll}
1 & 0 \\
1 & 1
\end{array}\right), B=\left(\begin{array}{ll}
1 & 1 \\
0 & 1
\end{array}\right), C=\left(\begin{array}{ll}
1 & 1 \\
0 & 1
\end{array}\right), D=\left(\begin{array}{ll}
1 & 0 \\
1 & 1
\end{array}\right) \\
& M=\left[\begin{array}{l}
\left(\begin{array}{ll}
1 & 0 \\
1 & 1
\end{array}\right)\left(\begin{array}{ll}
1 & 1 \\
0 & 1
\end{array}\right) \\
\left(\begin{array}{ll}
1 & 1 \\
0 & 1
\end{array}\right)\left(\begin{array}{ll}
1 & 0 \\
1 & 1
\end{array}\right)
\end{array}\right] \\
& (M / A)=\left(\begin{array}{cc}
1 & -1 \\
2 & 1
\end{array}\right),(M / B)=\left(\begin{array}{cc}
1 & 2 \\
-1 & 1
\end{array}\right), \\
& (M / C)=\left(\begin{array}{cc}
1 & 2 \\
-1 & 1
\end{array}\right),(M / D)=\left(\begin{array}{cc}
1 & -1 \\
2 & 1
\end{array}\right), \\
& S=\left(\begin{array}{ll}
(M / A) & (M / B) \\
(M / C) & (M / D)
\end{array}\right) \\
& S=\left(\begin{array}{l}
\left(\begin{array}{cc}
1 & -1 \\
2 & 1
\end{array}\right)\left(\begin{array}{cc}
1 & 2 \\
-1 & 1
\end{array}\right) \\
\left(\begin{array}{cc}
1 & 2 \\
-1 & 1
\end{array}\right)\left(\begin{array}{cc}
1 & -1 \\
2 & 1
\end{array}\right)
\end{array}\right) \quad K=\left(\begin{array}{ll}
\left(\begin{array}{ll}
1 & 0 \\
0 & 1
\end{array}\right)\left(\begin{array}{ll}
0 & 0 \\
0 & 0
\end{array}\right) \\
\left(\begin{array}{ll}
0 & 0 \\
0 & 0
\end{array}\right)\left(\begin{array}{ll}
1 & 0 \\
0 & 1
\end{array}\right)
\end{array}\right) \\
& V=\left(\begin{array}{ll}
\left(\begin{array}{ll}
0 & 0 \\
0 & 0
\end{array}\right)\left(\begin{array}{ll}
0 & 1 \\
1 & 0
\end{array}\right) \\
\left(\begin{array}{ll}
0 & 1 \\
1 & 0
\end{array}\right)\left(\begin{array}{ll}
0 & 0 \\
0 & 0
\end{array}\right)
\end{array}\right) \quad K V=\left(\begin{array}{l}
\left(\begin{array}{ll}
0 & 0 \\
0 & 0
\end{array}\right)\left(\begin{array}{ll}
0 & 1 \\
1 & 0
\end{array}\right) \\
\left(\begin{array}{ll}
0 & 1 \\
1 & 0
\end{array}\right)\left(\begin{array}{ll}
0 & 0 \\
0 & 0
\end{array}\right)
\end{array}\right) \\
& \text { Now } K V S=\left(\begin{array}{cc}
\left(\begin{array}{cc}
-1 & 1 \\
1 & 2
\end{array}\right)\left(\begin{array}{cc}
2 & 1 \\
1 & -1
\end{array}\right) \\
\left(\begin{array}{cc}
2 & 1 \\
1 & -1
\end{array}\right)\left(\begin{array}{cc}
-1 & 1 \\
1 & 2
\end{array}\right)
\end{array}\right) \text {, }
\end{aligned}
$$

$K V S$ is symmetric of rank 3 $\Rightarrow K V S$ is con-EP $\Rightarrow \mathrm{S}$ is con-s-k-EP.

$[S /(M / C)]=(M / B)-(M / D)(M / C)^{-1}(M / A)$ $(M / A)=\left(\begin{array}{cc}1 & -1 \\ 2 & 1\end{array}\right), \quad(M / B)=\left(\begin{array}{cc}1 & 2 \\ -1 & 1\end{array}\right)$ 


$$
\begin{aligned}
& (M / D)=\left(\begin{array}{cc}
1 & -1 \\
2 & 1
\end{array}\right), \\
& (M / C)^{-1}=\frac{1}{3}\left(\begin{array}{cc}
1 & -2 \\
1 & 1
\end{array}\right) \\
& {[S /(M / C)]=\left(\begin{array}{ll}
3 & 3 \\
0 & 3
\end{array}\right)}
\end{aligned}
$$

Hence $K_{2} v[S /(M / C)]=\left(\begin{array}{ll}0 & 3 \\ 3 & 3\end{array}\right)$ is con-EP,

that is $[S /(M / C)]$ is con-s- $\mathrm{k}_{2}-\mathrm{EP}$.

Also, $(M / C)=\left(\begin{array}{cc}1 & 2 \\ -1 & 1\end{array}\right) \Rightarrow K_{1} v(M / C)=\left(\begin{array}{cc}-1 & 1 \\ 1 & 2\end{array}\right)$ is con-EP. $K_{1} v(M / C)$ is con-EP $\Rightarrow(M / C)$ is con-s-k $\mathrm{k}^{-}$ EP.

Moreover $N(M / C) \subseteq N(M / A)$ and

$N(M / D)^{T} \subseteq N(M / C)^{T}$. But

$N[S /(M / D)] \subseteq N(M / D)$ and

$N[S /(M / C)]^{T} \subseteq N(M / A)^{T}$.

Further

$\left.K V\left(\begin{array}{cc}(M / C) & 0 \\ (M / A) & {[S /(M / C)}\end{array}\right]\right)=\left[\frac{\left(\begin{array}{cc}1 & 2 \\ -1 & 1\end{array}\right)}{\left(\begin{array}{cc}1 & -1 \\ 2 & 1\end{array}\right)} \frac{\left(\begin{array}{ll}0 & 0 \\ 0 & 0\end{array}\right)}{\left(\begin{array}{ll}3 & 3 \\ 0 & 3\end{array}\right)}\right]$ is not

con-EP.

Therefore,

$\left(\begin{array}{cc}(M / C) & (M / D) \\ 0 & {[S /(M / C)]}\end{array}\right)$ is not con-s-k-EP.

Thus the Theorem 2.5 and the Theorem 2.7 as well as the corollary 2.8 fail.

\section{Remarks 2.10}

We conclude from Theorem 2.5 and Theorem 2.7 that for a con-s-k-EP matrix of the form 2.2 and $k=k_{1} k_{2}$ where $K=\left(\begin{array}{cc}\mathrm{k}_{1} & 0 \\ 0 & \mathrm{k}_{2}\end{array}\right)$ and $v=\left(\begin{array}{ll}0 & v \\ v & 0\end{array}\right)$ the following are equivalent.

$$
\begin{aligned}
& N(M / C) \subseteq N(M / A), \\
& N[S /(M / C)] \subseteq N(M / D) \\
& N(M / C)^{T} \subseteq N(M / D)^{T}, \\
& N[S /(M / C)]^{T} \subseteq N(M / A)^{T}
\end{aligned}
$$

However this fails if we omit the condition that $S$ is con-s-k-EP.

For example,
Let $M=\left(\begin{array}{ll}A & B \\ C & D\end{array}\right)$, where

$A=\left(\begin{array}{ll}1 & 1 \\ 0 & 1\end{array}\right), \quad B=\left(\begin{array}{ll}0 & 1 \\ 1 & 0\end{array}\right), \quad C=\left(\begin{array}{ll}1 & 0 \\ 0 & 1\end{array}\right), \quad D=\left(\begin{array}{ll}1 & 1 \\ 0 & 1\end{array}\right)$

$M=\left[\frac{\left(\begin{array}{ll}1 & 1 \\ 0 & 1\end{array}\right)}{\left(\begin{array}{ll}1 & 0 \\ 0 & 1\end{array}\right)} \frac{\left(\begin{array}{ll}0 & 1 \\ 1 & 0\end{array}\right)}{\left(\begin{array}{ll}1 & 1 \\ 0 & 1\end{array}\right)}\right]$

$A, B, C, D \Rightarrow(M / A)=\left(\begin{array}{ll}0 & 1 \\ 1 & 0\end{array}\right)$,

$(M / B)=\left(\begin{array}{cc}0 & -2 \\ -1 & 0\end{array}\right), \quad(M / C)=\left(\begin{array}{cc}-1 & -1 \\ 1 & -1\end{array}\right)$,

$(M / D)=\left(\begin{array}{cc}1 & 0 \\ -1 & 2\end{array}\right)$

$S=\left(\begin{array}{ll}(M / A) & (M / B) \\ (M / C) & (M / D)\end{array}\right)$

$S=\left[\frac{\left(\begin{array}{ll}0 & 1 \\ 1 & 1\end{array}\right)}{\left(\begin{array}{cc}-1 & -1 \\ 1 & -1\end{array}\right)} \frac{\left(\begin{array}{cc}0 & -2 \\ -1 & 0\end{array}\right)}{\left(\begin{array}{cc}1 & 0 \\ -1 & 2\end{array}\right)}\right]$

$\left.K=\left[\frac{\left(\begin{array}{ll}1 & 0 \\ 0 & 1\end{array}\right)}{\left(\begin{array}{ll}0 & 0 \\ 0 & 0\end{array}\right)} \frac{\left(\begin{array}{ll}0 & 0 \\ 0 & 0\end{array}\right)}{\left(\begin{array}{ll}1 & 0 \\ 0 & 1\end{array}\right)}\right] \quad V=\frac{\left(\begin{array}{ll}0 & 0 \\ 0 & 0\end{array}\right)}{\left(\begin{array}{ll}0 & 1 \\ 1 & 0\end{array}\right)} \frac{\left(\begin{array}{ll}0 & 1 \\ 1 & 0\end{array}\right)}{\left(\begin{array}{ll}0 & 0 \\ 0 & 0\end{array}\right)}\right]$

$K V S=\left[\frac{\left(\begin{array}{cc}1 & -1 \\ -1 & -1\end{array}\right)}{\left(\begin{array}{ll}1 & 0 \\ 0 & 1\end{array}\right)} \frac{\left(\begin{array}{cc}-1 & 2 \\ 1 & 0\end{array}\right)}{\left(\begin{array}{cc}-1 & 0 \\ 0 & -2\end{array}\right)}\right]$ is not con-EP.

Therefore $S$ is not con-s-k-EP.

Here $K_{1} v(M / C)=\left(\begin{array}{cc}1 & -1 \\ -1 & -1\end{array}\right)$ is con-EP.

$\Rightarrow(M / C)$ is con-s-k-EP.

$$
\begin{aligned}
& K_{1} v(M / D) \neq\left(K_{1} v(M / D)\right)^{T}, \\
& K_{1} v(M / D) \neq\left((M / D)^{T} v K_{1}\right)^{T}, \\
& (M / D) \neq v K_{1} A^{T} v K_{1}, \\
& v(M / C) \subseteq v(M / A),
\end{aligned}
$$

and $v(M / C)^{T} \subseteq v(M / D)^{T}$.

Hence $[S /(M / C)]$ is independent of the choice of $(M / C)^{-}$.

Now 
$[S /(M / C)]=(M / B)-(M / A)(M / C)^{\dagger}(M / D)$

$(M / B)=\left(\begin{array}{cc}0 & -2 \\ -1 & 0\end{array}\right),(M / A)=\left(\begin{array}{ll}0 & 1 \\ 1 & 0\end{array}\right)$,

$(M / D)=\left(\begin{array}{cc}1 & 0 \\ -1 & 2\end{array}\right),(M / C)^{-1}=\frac{1}{2}\left(\begin{array}{cc}-1 & -1 \\ -1 & -1\end{array}\right)$

$[S /(M / C)]=\left(\begin{array}{cc}0 & -1 \\ -1 & 1\end{array}\right)$

$K_{2} v[S /(M / C)]=\left(\begin{array}{cc}-1 & 1 \\ 0 & -1\end{array}\right)$ is not con-EP.

$\Rightarrow[S /(M / C)]$ is not con-s-k $\mathrm{k}_{2}$-EP.

Also, $N[S /(M / C)]^{T} \subseteq N(M / D)^{T}$. But

$N[S /(M / C)] \not \subset N(M / D)$.

Thus, 2.12 holds while 2.11 fails.

\section{Remark 2.13}

It is clear by Remark 2.10 that for a con-s-k-EP martrix $S$, formula 2.6 gives $(K V S)^{\dagger}$ if and only if either 2.11 or 2.12 holds.

\section{Corollary 2.14}

Let $S$ be a matrix of the form 2.2 with $K$ and $V$ are of the forms 2.3 and 2.4 respectively, for which $(K V S)^{\dagger}$ is given by the formula then $S$ is con-s-k-EP if and only if both $(M / C)$ and $[S /(M / C)]$ and con-s-k-EP.

\section{Proof}

This follows from Theorem 2.5 and using Remark 2.13. Now we proceed to prove the most important Theorem.

\section{Theorem 2.15}

Let $S$ be of the form 2.2 with $\rho(S)=\rho(M / C)=r$. Then $S$ is con-s-k-EP and $K$ and $V$ are of the form 2.3 and 2.4 if and only if $(M / C)$ is con-s-k $\mathrm{k}_{1}-\mathrm{EP}_{\mathrm{r}}$ and $(M / A)(M / C)^{\dagger} v K_{1}=\left((M / C)^{\dagger}(M / D) v K_{2}\right)^{T}$.

\section{Proof}

Let $S$ be of the form 2.2 and let $\mathrm{k}=\mathrm{k}_{1} \mathrm{k}_{2}$ with $K=\left(\begin{array}{cc}k_{1} & 0 \\ 0 & k_{2}\end{array}\right)$ and $v=\left(\begin{array}{ll}0 & v \\ v & 0\end{array}\right)$ then $K V S=\left(\begin{array}{ll}K_{1} v(M / C) & K_{1} v(M / D) \\ K_{2} v(M / A) & K_{2} v(M / B)\end{array}\right)$.

Since $\rho(S)=\rho(M / C)=r$, $\rho(K V S)=\rho\left(K_{1} v(M / C)\right)=r$ by [ 6] $N(M / C)=N(M / A), N(M / C)^{T} \subseteq N(M / D)^{T}$ and $\left(K V S / K_{1} v(M / C)\right)$ $=K_{2} v[S /(M / C)]=0 \Rightarrow[S /(M / C)]=0$.

By Theorem 1.1 these relation equivalent to $K_{2} v(M / A)=K_{2} v(M / A)(M / C)$,

$K_{1} v(M / D)=K_{1} v(M / C)(M / C)^{\dagger}(M / D)$ and $K_{2} v(M / B)=K_{2} v(M / A)(M / C)^{\dagger}(M / D)$

Let us consider the matrices

$$
\begin{aligned}
P & =\left(\begin{array}{cc}
I & (M / A)(M / C) \\
0 & I
\end{array}\right) \\
Q & =\left(\begin{array}{ll}
I & (M / C)^{\dagger}(M / D) \\
0 & I
\end{array}\right) \text { and } L=\left(\begin{array}{cc}
0 & 0 \\
(M / C) & 0
\end{array}\right)
\end{aligned}
$$

$$
\begin{aligned}
& K V P L Q=\left(\begin{array}{cc}
K_{1} & 0 \\
0 & K_{2}
\end{array}\right)\left(\begin{array}{ll}
0 & v \\
v & 0
\end{array}\right)\left(\begin{array}{cc}
I & (M / A)(M / C)^{\dagger} \\
0 & I
\end{array}\right)\left(\begin{array}{cc}
0 & 0 \\
(M / C) & 0
\end{array}\right)\left(\begin{array}{cc}
I & (M / C)^{\dagger}(M / D) \\
0 & I
\end{array}\right) \\
& =\left(\begin{array}{cc}
0 & K_{1} v \\
K_{2} v & 0
\end{array}\right)\left(\begin{array}{cc}
(M / A)(M / C)^{\dagger}(M / C) & 0 \\
(M / C) & 0
\end{array}\right)\left(\begin{array}{cc}
I & (M / C)^{\dagger}(M / D) \\
0 & I
\end{array}\right) \\
& =\left(\begin{array}{cc}
0 & K_{1} v \\
K_{2} v & 0
\end{array}\right)\left(\begin{array}{rr}
(M / A)(M / C)(M / C)^{\dagger} & (M / A)(M / C)(M / C)^{\dagger}(M / C)^{\dagger}(M / C) \\
(M / C) & (M / C)(M / C)^{\dagger}(M / D)
\end{array}\right) \\
& =\left(\begin{array}{cc}
K_{1} v(M / C) & K_{1} v(M / C)(M / C)^{\dagger}(M / D) \\
K_{2} v(M / A)(M / C)(M / C)^{\dagger} & K_{2} v(M / A)(M / C)^{\dagger}(M / D)
\end{array}\right) \\
& =\left(\begin{array}{cc}
K_{1} v(M / C) & K_{1} v(M / D) \\
K_{2} v(M / A) & K_{2} v(M / B)
\end{array}\right) \\
& =\left(\begin{array}{cc}
K_{1} & 0 \\
0 & K_{2}
\end{array}\right)\left(\begin{array}{ll}
0 & v \\
v & 0
\end{array}\right)\left(\begin{array}{ll}
(M / A) & (M / B) \\
(M / C) & (M / D)
\end{array}\right) \\
& =K V S
\end{aligned}
$$


Thus $K V S$ can be factorized as $K V S=K V P L Q$. Since $K V P=(K V Q)^{\mathrm{T}}$.

We have $K V P^{T} V K=Q$. Therefore,

$K V S=K V P L K V P^{T} V K$

$=(K V P)(L K V)(K V P)^{T}$

$=(K V P)(K V L)(K V P)^{T}$

[since $L V K=K V L$ ].

Since $(M / C)$ is con-s- $k_{1}-\mathrm{EP}_{\mathrm{r}}$. We have $\mathrm{k}_{1} v(M / C)$ is con-EP ${ }_{\mathrm{r}}$.

Therefore $N(L)=N\left(L^{T} V K\right)$

(Theorem 2.11 of [1])

$\Rightarrow N(K V L)=N(K V L)^{T}$

By Theorem 1.3

$N\left[(K V P)(K V L)(K V P)^{T}\right]=N\left[(K V P)(K V L)^{T}(K V P)^{T}\right]$

$\Rightarrow N(K V S)=N\left[(K V S)^{T}\right]$

$\Rightarrow N(S)=N\left[S^{T} V K\right]$

$\Rightarrow S$ is con-s-k-EP (Theorem 2.11 of [1]).

Since $\rho(S)=r, S$ is con-s-k-EP .

Conversely, let us assume that $S$ is con-s-k-EP ${ }_{\mathrm{r}}$.

Since $S$ is con-s-k-EP,$K V S$ is con- $\mathrm{EP}_{\mathrm{r}}$. Since $K V S=$ $K V P L Q$, one choice of

$(K V S)^{-}=Q^{-1}\left(\begin{array}{cc}0 & 0 \\ (M / C)^{\dagger} & 0\end{array}\right) P^{-1} V K K V S$ is con-EP

$\Rightarrow N(K V S)=N\left[(K V S)^{T}\right]$ By Theorem 1.1

$(K V S)^{T}=(K V S)^{T}(K V S)^{-}(K V S)$.

That is,

$\left(\begin{array}{ll}K_{1} v(M / C) & K_{1} v(M / D) \\ K_{2} v(M / A) & K_{2} v(M / B)\end{array}\right)^{T}$

$=\left(\begin{array}{ll}K_{1} v(M / C) & K_{1} v(M / D) \\ K_{2} v(M / A) & K_{2} v(M / B)\end{array}\right)^{T}$

$Q^{-1}\left(\begin{array}{cc}0 & 0 \\ (M / C)^{\dagger} & 0\end{array}\right)$

$P^{-1} V K\left(\begin{array}{ll}K_{1} v(M / C) & K_{1} v(M / D) \\ K_{2} v(M / A) & K_{2} v(M / B)\end{array}\right)$

As the equation (at the bottom of this page). or conversely,

$\left(K_{1} v(M / C)\right)^{T}=\left(K_{1} v(M / C)\right)^{T}(M / C)^{\dagger}(M / C)$ and $\left(K_{2} v(M / C)\right)^{T}=\left(K_{1} v(M / C)\right)^{T}(M / C)^{\dagger}(M / D)$

From $\left(K_{1} v(M / C)\right)^{T}=\left(K_{1} v(M / C)\right)^{T}(M / C)^{\dagger}(M / C)$ it follows that

$$
\begin{aligned}
& N(M / C)=N\left[\left(K_{1} v(M / C)\right)^{T}\right] \\
& \Rightarrow N(M / C) \subseteq N(M / C)^{T} v K_{1} \Rightarrow(M / C)
\end{aligned}
$$

is con-s-k-EP.

Since $\rho(M / C)=r .(M / C)$ is con-s-k-EP .

From

$\left(K_{2} v(M / A)\right)^{T}=\left(K_{1} v(M / C)\right)^{T}(M / C)^{\dagger}(M / D)$

it follows that.

Now,

$$
\begin{aligned}
& K_{2} v(M / A)(M / C)^{\dagger} \\
& =(M / D)^{T}\left((M / C)^{\dagger}\right)^{T}\left(K_{1} v(M / C)\right)(M / C)^{\dagger} \\
& =(M / D)^{T}\left((M / C)^{\dagger}\right)^{T}\left((M / C)^{\dagger}(M / C) K_{1} v\right) \\
& =(M / D)^{T}\left[(M / C)^{\dagger}(M / C)(M / C)^{\dagger}\right]^{T}\left(v K_{1}\right)^{T} \\
& =(M / D)^{T}\left[(M / C)^{\dagger}\right]^{T}\left(v K_{1}\right)^{T} \\
& =\left[K_{1} v(M / C)^{\dagger}(M / D)\right]^{T}
\end{aligned}
$$

(By theorem 2.11 [1])

$$
\begin{aligned}
& K_{2} v(M / A)(M / C)^{\dagger}=\left[(M / C)^{\dagger}(M / D)\right]^{T} v K_{1} \\
& (M / A)(M / C)^{\dagger} v K_{1}=K_{2} v\left[(M / C)^{\dagger}(M / D)\right]^{T} \\
& (M / A)(M / C)^{\dagger} v K_{1}=\left[(M / C)^{\dagger}(M / D) v K_{2}\right]^{T}
\end{aligned}
$$

\section{Mark 2.16}

When $(M / A)$ is non singlular, $K V(M / A)$ is automatically con- $\mathrm{EP}_{\mathrm{r}}$ and $(M / A)$ is con-s-k-EP $\mathrm{P}_{\mathrm{r}}$ and Theorem 2.15 reduces to the following.

Corollary 2.17

Let $S$ be of the form 2.2 with $C$ non singular and $\rho[S]=\rho(M / C)$. Then $S$ is con-s-k-EP with $\mathrm{K}=\mathrm{k}_{1} \mathrm{k}_{2}$

$$
\begin{aligned}
\text { and } & v=\left(\begin{array}{ll}
0 & v \\
v & 0
\end{array}\right) \Leftrightarrow(M / A)(M / C)^{\dagger} v K_{1} \\
& =\left[(M / C)^{\dagger}(M / D) v K_{2}\right]^{T}
\end{aligned}
$$

$$
\left(\begin{array}{ll}
\left(K_{1} v(M / C)\right)^{T} & \left(K_{1} v(M / A)\right)^{T} \\
\left(K_{2} v(M / D)\right)^{T} & \left(K_{2} v(M / B)\right)^{T}
\end{array}\right)=\left(\begin{array}{ll}
\left(K_{1} v(M / C)\right)^{T}(M / C)^{\dagger}(M / C) & \left(K_{1} v(M / C)\right)^{T}(M / C)^{\dagger}(M / D) \\
\left(K_{1} v(M / D)\right)^{T}(M / C)^{\dagger}(M / C) & \left(K_{1} v(M / C)\right)^{T}(M / C)^{\dagger}(M / D)
\end{array}\right)
$$




\section{Remark 2.18}

When $\mathrm{k}(\mathrm{i})=\mathrm{i}$, we have $\mathrm{K}_{1}=\mathrm{K}_{2}=\mathrm{I}$, then the Theorem 2.15 reduces to the result for con-s-EP matrices.

When KV = I then Theorem 2.15 reduces to Theorem 3 of [5].

\section{Remark 2.19}

Theorem 2.15 fails if we relax the condition on the rank of $S$.

For example, let us consider the matrix $S$ and $K$ given in Remark 2.10, $\rho[K V S]=\rho[S]=2$.

But $\rho\left(K_{1} V(M / C)\right)=\rho(M / C)=1$,

$\rho(K V S) \neq \rho\left(K_{1} v(M / A)\right) \Rightarrow \rho(S) \neq \rho(M / A)$.

$K V S$ is not con-EP

Therefore $S$ is not Con-s-k-EP.

However,

$$
\begin{aligned}
K_{1} V(M / C) & =\left(\begin{array}{ll}
1 & 0 \\
0 & 1
\end{array}\right)\left(\begin{array}{ll}
0 & 1 \\
1 & 0
\end{array}\right)\left(\begin{array}{cc}
-1 & -1 \\
1 & -1
\end{array}\right) \\
& =\left(\begin{array}{ll}
0 & 1 \\
1 & 0
\end{array}\right)\left(\begin{array}{cc}
-1 & -1 \\
1 & -1
\end{array}\right)=\left(\begin{array}{cc}
1 & -1 \\
-1 & -1
\end{array}\right) \text { is con-EP. }
\end{aligned}
$$

Therefore $(M / C)$ is con-s- $\mathrm{k}_{1}-\mathrm{EP}$ and

$$
\begin{aligned}
& (M / C)^{-1}=\frac{1}{2}\left(\begin{array}{ll}
-1 & -1 \\
-1 & -1
\end{array}\right), \\
& (M / A)(M / C)^{-1} v K_{1}=\frac{1}{2}\left(\begin{array}{ll}
-1 & -1 \\
-1 & -1
\end{array}\right), \\
& (M / C)^{-1}(M / D) v K_{2}=\left(\begin{array}{ll}
-1 & 0 \\
-1 & 0
\end{array}\right) .
\end{aligned}
$$

Thus the theorem fails.

\section{Corollary $\mathbf{2 . 2 0}$}

Les $S$ be a $2 \mathrm{r} \times 2 \mathrm{r}$ matrix of rank $\mathrm{r}$. Thus $S$ is con-s-k-EP $\mathrm{r}_{\mathrm{r}}$ with $\mathrm{K}=\mathrm{K}_{1} \mathrm{~K}_{2}$, where

$\left(\begin{array}{cc}K_{1} & 0 \\ 0 & K_{2}\end{array}\right)$ and $V=\left(\begin{array}{cc}v & 0 \\ 0 & v\end{array}\right) \Leftrightarrow$ every secondary sub matrix of $S$ of rank r is con-s-k-EP .

\section{Proof}

Suppose $S$ is con-s-k-EP $\mathrm{P}_{\mathrm{r}}$ matrix then $K V S$ is an con-EP $\mathrm{P}_{\mathrm{r}}$ matrix by Theorem 2.11 [1]. Let $K_{1} v(M / C)$ be any Principal submatrix of $K V S$ such that $\rho[K V S]=\rho\left[K_{1} v(M / C)\right]=r$, then there exists a permutation matrix $P$ such that,

$$
(K V S)^{T}=P(K V S) P^{T}\left(\begin{array}{ll}
K_{1} v(M / C) & K_{1} v(M / D) \\
K_{2} v(M / A) & K_{2} v(M / B)
\end{array}\right)
$$

with $\rho[K V S]=\rho\left[K_{1} v(M / C)\right]=r$. By [4] $[K V S]^{T}$ is con-EP . Now we conclude from Theorem 2.15 that $\left(K_{1} v(M / C)\right)$ is con-EP $\mathrm{E}_{\mathrm{r}}$ That is $(M / C)$ is con-s- $\mathrm{k}_{1}-\mathrm{EP}_{\mathrm{r}}$ Since $[M / C]$ is arbitrary it follows that every secondary submatrix of rank $\mathrm{r}$ is con-s-k-EP . The converse is obvious.

The conditions under which a partitioned matrix is decomposed into complementary sum and $S$ of con-sk-EP matrices are given. $S_{1}$ and $S_{2}$ and called complementary summands of $S$ if

$S=S_{1}+S_{2}$ and $\rho[S]=\rho\left[S_{1}\right]+\rho\left[S_{2}\right]$.

\section{Theorem 2.21}

Let $S$ be of the form 2.2 with

$\rho(S)=\rho(M / C)+\rho[S /(M / C)]$,

where $[S /(M / C)]=(M / B)-(M / A)\left((M / C)^{\dagger}(M / D)\right.$

and $K$ is of the form 2.3 and $V$ is of the form 2.4. If $(M / C)$ is con-s- $\mathrm{k}_{1}$-EP and $[S /(M / C)]$ is con-s- $\mathrm{k}_{2}$-EP matrices such that

$$
\begin{aligned}
& (M / A)(M / C)^{\dagger} v K_{1}=\left((M / C)^{\dagger}(M / D) v K_{2}\right)^{T} \text { and } \\
& (M / D)[S /(M / C)]^{\dagger} v K_{2}=\left([S /(M / C)]^{\dagger}(M / C) v K_{1}\right)^{T}
\end{aligned}
$$

then $S$ can be decomposed into complementary summands of con-s-k-EP matrices.

\section{Proof}

Let us consider the matrices,

$$
S_{1}=\left(\begin{array}{cc}
(M / C) & (M / C)(M / C)^{\dagger}(M / D) \\
(M / A)(M / C)^{\dagger}(M / C) & (M / A)(M / C)^{\dagger}(M / D)
\end{array}\right)
$$

and

$$
S_{2}=\left(\begin{array}{cc} 
& \left(I-(M / C)(M / C)^{\dagger}\right) \\
0 & (M / D) \\
(M / A) & {[S /(M / C)]}
\end{array}\right) .
$$

Taking into account that

$$
\begin{aligned}
& (M / C) \subseteq N\left((M / A)(M / C)^{\dagger}(M / A)\right) \\
& N(M / C) v K_{1} \subseteq N\left((M / A)(M / C)^{\dagger}(M / C)\right)^{T} v K_{1} \text { and }
\end{aligned}
$$

$$
\begin{aligned}
& {\left[S_{1} /(M / C)\right]=(M / A)(M / C)^{\dagger}(M / D)-\left((M / A)(M / C)^{\dagger}(M / D)\right)(M / C)^{-}\left((M / C)(M / C)^{\dagger}(M / D)\right)_{1}} \\
& =(M / A)(M / C)^{\dagger}(M / D)-\left((M / A)(M / C)^{\dagger}\right)\left((M / C)(M / C)^{-}(M / C)\right)(M / C)^{\dagger}(M / D)_{1} \\
& =(M / A)(M / C)^{\dagger}(M / D)-(M / A)\left((M / C)^{\dagger}(M / C)(M / C)^{\dagger}\right)(M / D) \\
& =(M / A)(M / C)^{\dagger}(M / D)-(M / A)(M / C)^{\dagger}(M / D) \\
& =0
\end{aligned}
$$


We obtain by [6] that $\rho\left(S_{1}\right)=\rho(M / C)$. Since $(\mathrm{M} / \mathrm{C})$ is con-s-k $\mathrm{k}_{1}$-EP and $\left((M / A)(M / C)^{\dagger}(M / C)\right)(M / C)^{\dagger} v K_{1}$ $=(M / A)(M / C)^{\dagger}(M / C)(M / C)^{\dagger} v K_{1}$ $=(M / A)(M / C)^{\dagger} v K_{1}$ $=\left((M / C)^{\dagger}(M / D) v K_{1}\right)^{T}$ $=\left((M / C)^{\dagger}\left((M / C)(M / C)^{\dagger}(M / D)\right) v K_{2}\right)^{T}$

We have by Theorem 2.15, that is $S_{1}$ is con-s-k $\mathrm{k}_{1}$-EP. Since $\rho(S)=\rho(M / C)+\rho[S /(M / C)]$,

Theorem 1 of [6], gives

$$
N[S /(M / C)]=N\left(\left[I-(M / C)(M / C)^{\dagger}\right](M / D)\right),
$$$$
\left[I-(M / C)(M / C)^{\dagger}\right](M / D)[S /(M / C)]^{\dagger} v K_{2}
$$$$
=\left[I-(M / C)(M / C)^{\dagger}\right]\left[[S /(M / C)]^{\dagger}(M / A) v K_{1}\right]^{T}=\left[\left[[S /(M / C)]^{\dagger}(v / A) v K_{1}\right]\left[I-(M / C)(M / C)^{\dagger}\right]^{T}\right]^{T}
$$$$
=\left[[S /(M / C)]^{\dagger}(v / A)\left[\left[I-(M / C)(M / C)^{\dagger}\right] K_{1} v\right]^{T}\right]^{T}=\left[[S /(M / C)]^{\dagger}(v / A)\left[K_{1} v-(M / C)(M / C)^{\dagger} K_{1} v\right]^{T}\right]^{T}
$$$$
=\left[[S /(M / C)]^{\dagger}(M / A)\left[K_{1} v-K_{1} v\left((M / C)^{\dagger}(M / C)\right]^{T}\right]^{T}=\left[[S /(M / C)]^{\dagger}(M / A)\left[K_{1} v-I-(M / C)^{\dagger}(M / C)\right]^{T}\right]^{T}\right.
$$$$
=\left[[S /(M / C)]^{\dagger}(M / A) I-\left[(M / C)^{\dagger}(M / C)\right]^{T} v K_{1}\right]^{T}
$$

\section{REFERENCES}

[1] S. Krishnamoorthy, K. Gunasekaran and B. K. N. Muthugobal, "con-s-k-EP Matries,” Journal of Mathematical Sciences and Engineering Applications, Vol. 5, No. 1, 2011, pp. 353-364.

[2] C. R. Rao and S. K. Mitra, "Generalized Inverse of Matrices and Its Applications,” Wiley and Sons, New York, 1971.

[3] R. Penrose, "On Best Approximate Solutions of Linear Matrix Equations," Mathematical Proceedings of the Cambridge Philosophical Society, Vol. 52, No. 1, 1959, pp. 17-19.

[4] T. S. Baskett and I. J. Katz, "Theorems on Products of $E P_{r}$ Matrices," Linear Algebra and Its Applications, Vol. 2, No. 1, 1969, pp. 87-103.

$$
N[S /(M / C)]^{T}=N\left((M / C)\left[I-(M / C)^{\dagger}(M / C)\right]\right)^{T}
$$

and

$$
\begin{aligned}
& {\left[I-(M / C)(M / C)^{\dagger}\right](M / D)[S /(M / C)]^{\dagger}} \\
& \subset\left[I-(M / C)^{\dagger}(M / C)\right]=0
\end{aligned}
$$

Therefore, $\left[S_{2} /[S /(M / C)]\right]=0$.

Thus by [7] we get $\rho\left(S_{2}\right)=\rho[S /(M / C)]$. Thus $\rho(S)=\rho\left(S_{1}\right)+\rho\left(S_{2}\right)$.

Further using $=(M / C)(M / C) K_{1} v=K_{1} v(M / C)^{\dagger}(M / C)$

We obtain,
[5] A. R. Meenakshi, "On Schur Complements in an EP Matrix, Periodica, Mathematica Hungarica," Periodica Mathematica Hungarica, Vol. 16, No. 3, 1985, pp. 193200.

[6] D. H. Carlson, E. Haynesworth and T. H. Markham, “A Generalization of the Schur Complement by Means of the Moore-Penrose Inverse," SIAM Journal on Applied Mathematics, Vol. 26, No. 1, 1974, pp. 169-175.

[7] A. B. Isral and T. N. E. Greviue, "Generalized Inverses Theory and Applications,” Wiley and Sons, New York, 1974.

[8] S. Krishnamoorthy, K. Gunasekaran and B. K. N. Muthugobal, "On Sums of con-s-k-EP Matrix,” Thai Journal of Mathematics, in Press, 2012. 